\title{
Physics, biology and the right chemistry
} Patricia Bassereau ${ }^{1}$ and Bruno Goud ${ }^{2 *}$

\author{
Addresses: ${ }^{1}$ Department of Physico-Chemistry (UMR CNRS/Institut Curie 168), Institut Curie, 26 rue d'Ulm, 75248 Paris CEDEX 05, France; \\ ${ }^{2}$ Department of Cell Biology (UMR CNRS/Institut Curie 144), Institut Curie, 26 rue d'Ulm, 75248 Paris CEDEX 05, France \\ * Corresponding author: Bruno Goud (bruno.goud@curie.fr) \\ FI000 Biology Reports 20II, 3:7 (doi:10.34I0/B3-7) \\ This is an open-access article distributed under the terms of the Creative Commons Attribution-Non Commercial License \\ (http://creativecommons.org/licenses/by-nc/3.0/legalcode), which permits unrestricted use, distribution, and reproduction in any medium, \\ provided the original work is properly cited. You may not use this work for commercial purposes. \\ The electronic version of this article is the complete one and can be found at: http://fl $000 . c o m / r e p o r t s / b / 3 / 7$
}

\begin{abstract}
Joint studies that involve biologists and physicists are becoming more frequent and have contributed to the identification and understanding of physical parameters underlying key biological processes. Here, we illustrate the main findings resulting from a 10 -year collaboration between a cell biologist and an experimental physicist, both interested in the mechanisms of intracellular transport and membrane dynamics in eukaryotic cells.
\end{abstract}

\section{Introduction}

Intracellular exchange is the essence of cellular life. For prokaryotic cells, simple diffusion of nutrients and waste products suffices. But for eukaryotic cells, whose functional organization relies on an elaborate network of intracellular membranes (also called organelles), a complex mass transit system has evolved. It consists of membrane-bound structures called transport carriers, of vesicular or tubular shape, that carry cargo into, out of, and around the cell. Our active interest throughout the past decade has been in the interplay between biophysical properties of membranes and specific functions of biological molecules that give rise to in-cell transport systems.

When we were first introduced in 2000 by Jacques Prost, a theoretician physicist who was then the director of the Physics Department at the Curie Institute in Paris where we both worked, we could not have guessed that we'd become such close research collaborators, given our divergent interests and experience. Yet the encounter was no coincidence. The Institute was fostering links between cell biology and physics through a program called "Physics of the Cell" that allocated small crossdepartment grants. We quickly realized that the mechanisms behind the formation of transport carriers in cells excited us both, and enthusiastically accepted the funding and started our collaboration.
One of us (BG) was an immunologist and cell biologist by training. From 1986 onwards, Bruno focused on Rab proteins, a family of small GTPases that regulate intracellular transport and membrane trafficking. The year before meeting Patricia, Bruno's team, working with Ernst Stelzer's group at the European Molecular Biology Laboratory (EMBL) in Germany, had visualized the highly dynamic process that initiates and moves membrane tubules along microtubules from the Golgi to the cell periphery. This novel transport pathway is controlled by Rab6, a Golgi-associated Rab [1].

The other of us (PB) was trained as an experimental physicist in the field of "soft matter". Initially, Patricia worked on lyotropic smectic phases stabilized by thermal fluctuations. Taking into account the intrinsic nonequilibrium nature of biological membranes, this led her to study, in collaboration with Jacques Prost, the fluctuations of lipid membranes in the presence of active membrane proteins. Given a growing interest in membrane trafficking, Patricia had known of Bruno's stimulating findings on the membrane tubules decorated by Rab6 before we met.

\section{Assays and models}

Almost immediately, we agreed on our joint goal: we would develop an in-vitro assay that mimics the initial steps of intracellular transport. In particular, we wanted 
to capture membrane deformation and the creation of tubular carriers. We recruited a student, Aurélien Roux, to work with us. The idea was that Aurélien would attach biotinylated kinesins to the lipid membrane of biotinylated giant unilamellar vesicles, known as GUVs, by means of $100-\mathrm{nm}$ polystyrene beads coated with streptavidin (Figure 1, top). When incubated with microtubules and ATP in small chambers, GUVs did indeed give rise to membrane tubes and to complex tubular networks that could be visualized by confocal microscopy (Figure 2) [2]. This experiment was the first demonstration that the force generated by kinesins was sufficient to pull a membrane tube from a membrane reservoir. Remarkably, as shown by transmission electron microscopy, the tubes that were pulled from GUVs made of EPC (egg phosphatidylcholine) had a constant diameter of $40(+/-10 \mathrm{~nm})$, a value close to that estimated for tubular transport carriers in vivo.

A second student, Cécile Leduc, was able to monitor the dynamic accumulation of kinesins at the tips of membrane tubes [3], which were collectively responsible for the force generation. For these experiments, kinesins tagged with steptavidin were directly attached

Figure I. Formation of membrane nanotubes from giant unilamellar vesicles (GUVs) by molecular motors

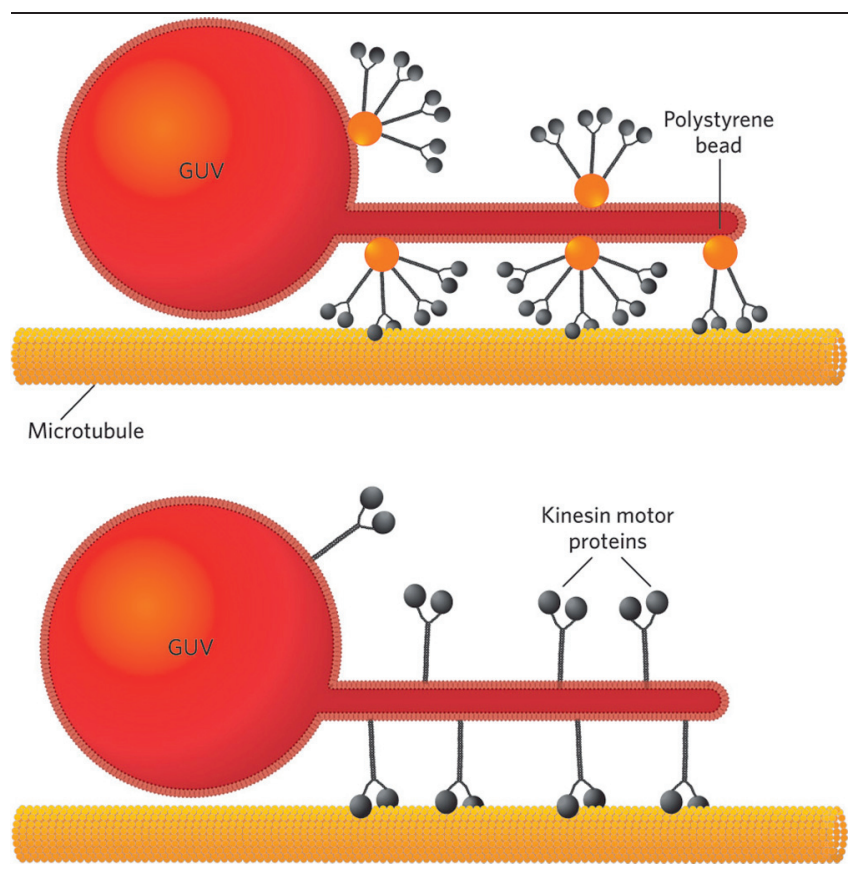

Membrane tubes similar to those involved in intracellular transport can be pulled by kinesin motor proteins bound to GUVs that move along immobilized microtubules in the presence of ATP. The kinesins can be bound either via small streptavidin-coated polystyrene beads (top) or via streptavidin molecules associated with the lipid bilayer itself (bottom). to the lipid membrane of GUVs via biotinylated lipids, following a method developed by the team of Marileen Dogterom in the Netherlands [4] (Figure 1, bottom). In parallel, colleagues in the Physics Department had started to work on theoretical aspects of the physics of membrane tubes. Their analysis of the dynamics of motors on both vesicle and tube surfaces fitted Cécile's experimental observations. Together, these studies identified the initial minimal surface density of motors on the vesicle, below which no membrane tubes can be pulled [3] and, conversely, a maximum membrane tension above which motors cannot pull tubes. Extrapolating this finding to cell biology, transport might be switched on and off in cells by regulating the number of available motors, the number of potential motor attachment sites (proteins or lipids) on the membrane, or the tension of the membrane.

\section{Getting physical}

Using this minimal model of the early steps of intracellular transport, we set out to investigate their physical parameters, including membrane curvature, membrane bending rigidity, and membrane tension.

Given the small diameter of the transport carriers inside the cells (typically 40-100 nm), they are highly curved structures in comparison with the membrane from which

Figure 2. Confocal image of a network of nanotubes formed from a giant unilamellar vesicle (GUV) containing fluorescent lipids and pulled by kinesin motors

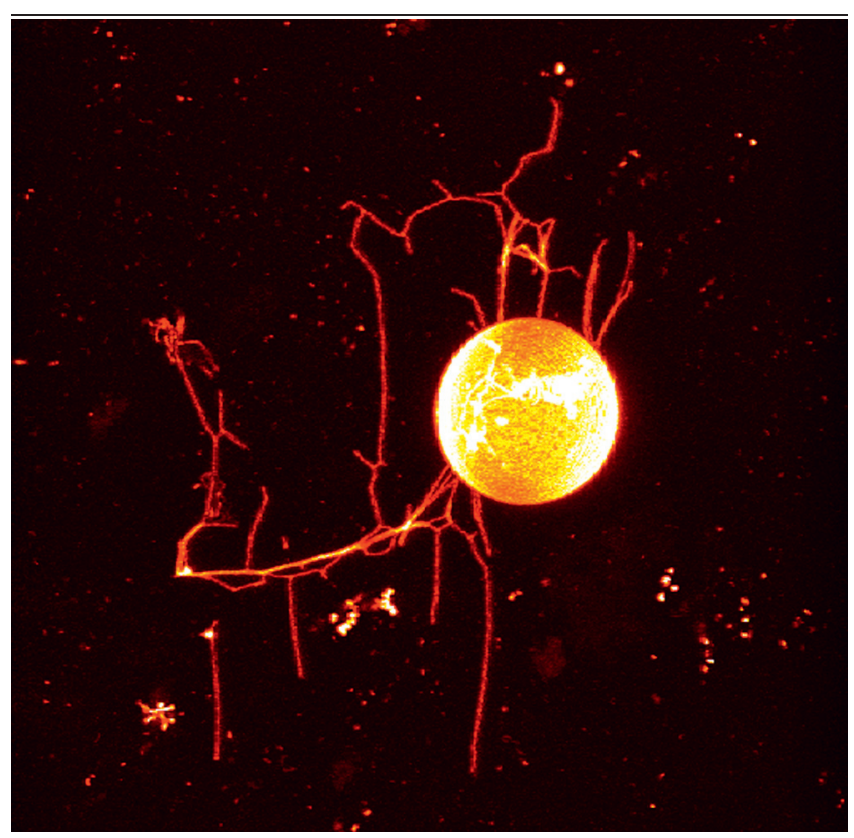

Image courtesy of C. Leduc. 
they originate, which can be viewed as "flat". During the early stages of vesicle formation, protein and lipid sorting occurs, ensuring efficient and accurate transport between cell compartments and the maintenance of membrane homeostasis. In 2004, the sorting of proteins had already been well described but lipid sorting was much less clearly understood. To investigate it, we pulled tubes from GUVs that were prepared from ternary mixtures of brain sphingomyelin, cholesterol, and dioleylphosphatidylcholine (DOPC), representing the three major lipid components of the external leaflet of the plasma membrane. Depending on the relative proportion of the three lipids, the lipids can be either mixed forming a single homogeneous phase, or they demix and preferentially segregate in different phases. In the latter, two phases coexist, a liquid disordered phase (Ld) enriched in DOPC, and a liquid ordered phase (Lo) enriched in cholesterol and sphingomyelin.

The force $(f)$ required to pull a tube is proportional to the square root of the bending rigidity $(\kappa)$ and of the membrane tension $(\sigma)(f=2 \pi \sqrt{2 \kappa \sigma})$. Using an optical tweezers setup coupled to a micropipette system (Figure 3), we measured the bending rigidity of the Lo and Ld phases. Membranes in the Lo phase are about twice as rigid as membranes in the Ld phase [6]. Given this, we predicted that lipids of the Lo phase should be excluded from the tubes, to reduce the energy cost to bend the membrane in the tube. This is exactly what we observed: in phase-separated vesicles, tubes were preferentially pulled out from the Ld phase; in homogeneous vesicles, the Lo lipids were depleted from the tubes. These experiments provided the first direct demonstration that lipid sorting can occur during the formation of highly curved membrane tubes [6].

Figure 3. General scheme of the experimental system used to study forces on a membrane

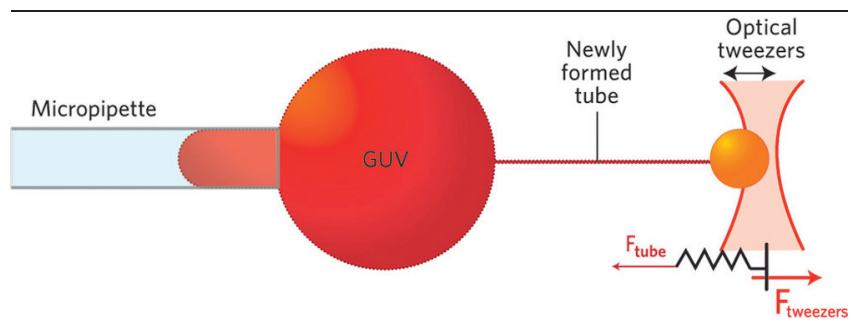

A membrane nanotube is pulled from a giant unilamellar vesicle (GUV) aspirated in a micropipette (left). A bead (orange sphere) trapped in optical tweezers is attached to the GUV. The tube is formed by pulling the micropipette away from the GUV. At equilibrium, the force required to pull a tube is calculated from the bead displacement and from the tweezers' stiffness calibration.
To measure lipid sorting in a quantitative way, another jointly supervised PhD student, Benoît Sorre, built a novel experimental setup that combined confocal microscopy, optical tweezers, and micropipette aspiration. From the force measurement and from the analysis of the redistribution of fluorescent lipids between the tube and GUV, he could show that lipid sorting was effective only when the lipid composition of the GUV was near phase separation. This was done by comparing lipid distribution in the tube and in the vesicle from GUVs made of different lipid compositions. Remarkably, this process was amplified in the presence of proteins that are able to cluster lipids, such as cholera toxin [7]. Our theoretician colleagues developed a model based on membrane elasticity and nonideal solution theory to explain Benoit's results. This model posits that the sorting of lipids between the tube and vesicle is determined by a trade-off between mixing entropy and bending energy. By excluding lipids that have a tendency to form more rigid membranes, the energy required to form a curved membrane and thus a thin tube, is lowered. However, due to the small size of the lipids, this effect is dominant over lipid mixing entropy only for compositions close to phase separation (or demixing) [7].

\section{Lipid-manipulating proteins}

Aurélien made another interesting observation: when phase separation of lipids occurred in the tubes, fission events took place at the boundary between Lo and Ld domains [6]. It turns out that these observations are consistent with a theoretical analysis in which membrane rupture was predicted to originate from the difference of elastic constants of the two phases and the resulting constricting line tension [8]. Since cell membranes are likely close to phase separation, these results raised the interesting hypothesis that the role of the numerous proteins implicated in sorting and fission events in vivo [9] could be to trigger phase separation in membrane lipids, either by clustering specific lipids or by inducing membrane tubulation.

Mechanoenzymes, including dynamin, are known to contribute to membrane fission. Dynamin is a large GTPase that has been shown to polymerize into a helical collar at the neck of endocytic buds, and induces the formation of endocytic vesicles through neck fission. Our work on line tension-induced membrane fission motivated us to explore the role of membrane curvature on the helical assembly of dynamin. Using a combination of confocal microscopy and optical tweezers, we discovered that membrane curvature triggers dynamin assembly, and thus the precise timing for the detachment of endocytic vesicles from the membrane [10]. 
The functions of proteins that sense or induce membrane curvature have received considerable attention recently because of the importance of this phenomenon during the formation of vesicles and tubular carriers. Vesicles are surrounded by coat proteins (e.g., COPI coatomer), which are recruited to the site by activated coatrecruitment proteins such as ADP-ribosylation factor 1 (Arf1). Several proteins involved in vesicle formation, including Arf GTPase-activating protein 1 (ArfGAP1), contain a structural motif, named the ArfGAP1 lipidpacking sensor (ALPS), that senses membrane curvature. This motif is a nonclassical amphipathic $\alpha$-helix, whose polar face, instead of being composed of positively charged amino acids, is enriched in serine and threonine residues [11], a feature that likely explains the extreme sensitivity of proteins with ALPS motifs to membrane curvature. As ArfGAP1 is a GTPase-activating protein, it stimulates GTP hydrolysis of GTP bound to Arf1, a small G protein located on Golgi membranes. Arf1 binds strongly in its GTP-bound conformation to membranes. When this happens, Arf1 promotes the assembly of the COPI coat on the surface of transport vesicles operating between Golgi and endoplasmic reticulum (see Figure 4 and Figure 5). Remarkably, the ArfGAP1 activity on Arf1 bound to small (highly curved) liposomes $(35 \mathrm{~nm})$ is about 500 times higher than on Arf1 bound to larger (more flat) liposomes $(150 \mathrm{~nm})$ [13]. Our assay system was ideal for studying the spatial distribution of proteins between curved and noncurved membrane regions. Ernesto Ambrioggio, a

Figure 4. Creation of a gradient of ADP-ribosylation factor I (ArfI) along a membrane tube connected to a giant unilamellar vesicle (GUV)

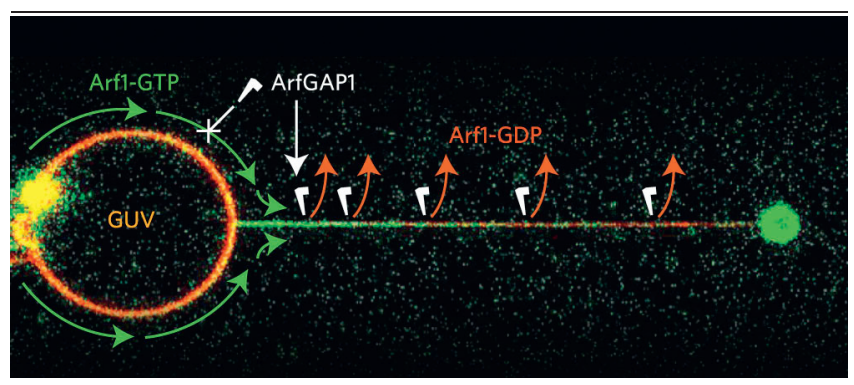

A gradient of Arfl molecules (green fluorescent label) is created along a membrane tube containing lipids (red fluorescent label) pulled using a bead (large green sphere, right) trapped in an optical tweezers. The gradient is due to the competition between diffusion of Arfl-GTP from the giant unilamellar vesicle (GUV) on the left into the pulled membrane tube (green arrows) and the dissociation of Arfl-GDP induced by ArfGAPI hydrolysis of Arfl-GTP, which occurs in the tube because of its high curvature. The low curvature of the GUV membrane prevents ArfGAPI binding and protects Arfl-GTP from hydrolysis. post-doc, worked with Benoît to compare the sensitivity to curvature of Arf1, an ALPS motif, and ArfGAP1. Arf1 bound almost equally well to the GUV membrane and to a tube pulled with kinesin motors or optical tweezers. Thus, Arf1 binding is, at most, only weakly sensitive to membrane curvature. In contrast, ALPS and ArfGAP1 did not bind to the GUV at all. A curvature threshold was found for their binding to the membrane tubes: almost no binding was detected on tubes with a radius above $35+/-5 \mathrm{~nm}$, whereas below this critical radius, ALPS and ArfGAP1 density on the membrane increased linearly [12]. The next step towards understanding the influence of membrane curvature on ArfGAP1 enzymatic activity was to investigate the distribution of Arf1 on the vesicle and the membrane tube in the presence of ArfGAP1. Remarkably, ArfGAP1-induced GTP hydrolysis was able to generate an Arf1 gradient along the tube, with Arf1 density decreasing linearly from the base to the tip of the membrane tube (Figure 4). This nonuniform distribution of Arf1 along the tube was suggestive of a diffusionreaction process: ArfGAP1 activity induces the dissociation of Arf1 from the tube; however, because the tube is connected to the vesicle (GUV), Arf1 can diffuse from the vesicle to the tube and this compensates for Arf1 dissociation. This diffusion-reaction model was experimentally validated [12]. These finding suggest that membrane fission is the triggering event for coat disassembly. When the neck of the COPI-coated vesicle is cut, the loss of Arf1 by GTP hydrolysis is no longer compensated for by Arf1-GTP diffusion. As a result, the coat should readily disassemble (Figure 5).

Recently, Benoît has used a similar approach to study amphiphysin, a protein with a crescent-shaped binding

Figure 5. Proposed model for the association and dissociation of COPI coatomer in vivo

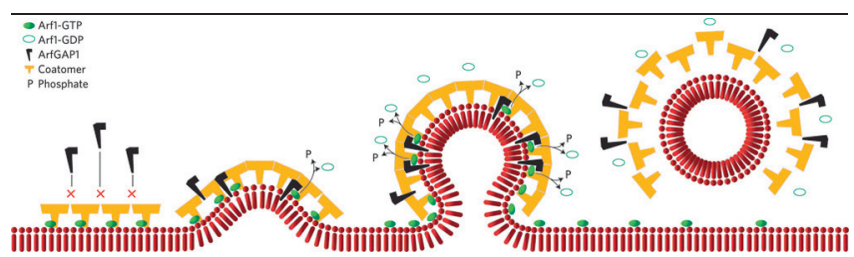

Coat proteins are recruited to the site of vesicle budding by membranebound ArfI in its GTP form, and begin to deform the donor membrane. Sensing membrane curvature, ArfGAPI is recruited to the budding site where it hydrolyzes GTP bound to Arfl, which then dissociates. As long as the budding site is attached to the donor membrane, the GTP form of ArfI is replenished at the budding site. Once dissociated, the new vesicle lacks a fresh supply of Arfl-GTP. After all the ArfI-GTP has been hydrolyzed by ArfGAPI, the COPI coat dissociates from the newly formed transport vesicle or tubule. 
domain called an N-BAR domain involved in the generation of clathrin-coated vesicles. He showed that this protein has a dual behavior: at low concentration, its level on the membrane depends on membrane curvature, similarly to ArfGAP1, but it cannot deform the membrane. At high concentration, amphiphysin can constrict a membrane tube, independently of the membrane tension (Sorre et al., submitted).

\section{Perspectives}

Our collaboration, along with our interactions with theoretical physicists, has been particularly fruitful and gratifying over the past 10 years. Right now we are planning to deepen our partnership still further with an ambitious project aimed at understanding how different classes of actin-based motors of the myosin family function in membrane trafficking and membrane dynamics. This project will exploit the minimal in-vitro system developed in our laboratories.

Over the last decade we have challenged one another, and generated reciprocal interests: Bruno has become more receptive and interested in physical concepts and Patricia continues to explore projects more related to cell biology. What is our advice to others that might be thinking of a cross-disciplinary collaboration? Go for it, the challenges and rewards of the alternative perspective will add a new dimension to you research.

\section{Abbreviations}

Arf1, ADP-ribosylation factor 1; ArfGAP1, ADP-ribosylation factor GTPase-activating protein 1; DOPC, dioleylphosphatidylcholine; GUV, giant unilamellar vesicle; Ld, liquid disordered; Lo, liquid ordered.

\section{Competing interests}

The authors declare that they have no competing interests.

\section{Acknowledgments}

We wish to thank present and past members of our teams for their numerous contributions.

\section{References}

I. White J, Johannes L, Mallard F, Girod A, Grill S, Reinsch S, Keller P, Tzschaschel B, Echard A, Goud B, Stelzer EH: Rab6 coordinates a novel Golgi to ER retrograde transport pathway in live cells. J Cell Biol 1999, 147:743-60.

2. Roux A, Cappello G, Cartaud J, Prost J, Goud B, Bassereau P: A minimal system allowing tubulation with molecular motors pulling on giant liposomes. Proc Natl Acad Sci U S A 2002, 99:5394-9.

3. Leduc $C$, Campàs $O$, Zeldovich $K B$, Roux $A$, Jolimaitre $P$, BourelBonnet L, Goud B, Joanny JF, Bassereau P, Prost J: Cooperative extraction of membrane nanotubes by molecular motors. Proc Natl Acad Sci U S A 2004, I01:17096-10I.

4. Koster G, VanDuijn M, Hofs B, Dogterom M: Membrane tube formation from giant vesicles by dynamic association of motor proteins. Proc Natl Acad. Sci U S A 2003, 100:15583-8.

5. Derényi I, Jülicher F, Prost J: Formation and interaction of membrane tubes. Phys Rev Lett 2002, 88:238I0I.

6. Roux A, Cuvelier D, Nassoy P, Prost J, Bassereau P, Goud B: Role of curvature and phase transition in lipid sorting and fission. EMBO J 2005, 24:1537-45.

FI000 Factor 13

Evaluated by Markus Engstler 06 Apr 2005, Anne Spang 04 May 2005, Gerrit van Meer 04 May 2005, David Stephens 10 May 2005

7. Sorre B, Callan-Jones A, Manneville JB, Nassoy P, Joanny JF, Prost J, Goud B, Bassereau P: Curvature-driven lipid sorting needs proximity to a demixing point and is aided by proteins. Proc Natl Acad Sci U S A 2009, 106:5622-6.

8. Allain J, Storm C, Roux A, Ben Amar M, Joanny JF: Fission of a multiphase membrane tube. Phys Rev Lett 2004, 93:158104.

9. Slepvev VI, De Camilli P: Accessory factors in clathrin-dependent synaptic vesicle endocytosis. Nat Rev Neurosci 2000, I:161-72.

10. Roux A, Koster G, Lenz M, Sorre B, Manneville JB, Nassoy P, Bassereau $P$ : Membrane curvature controls dynamin polymerization. Proc Natl Acad Sci U S A 2010, 107:4I4I-6.

II. Drin G, Casella JF, Gautier R, Boehmer T, Schwartz TU, Antonny B: A general amphipathic alpha-helical motif for sensing membrane curvature. Nat Struct Mol Biol 2007, 14:138-46.

FI000 Factor 12

Evaluated by Roger Williams 08 Mar 2007, Xiayang Qiu 20 Mar 2007, Edwin Chapman 16 May 2007, Alfred Wittinghofer 05 Jun 2007

12. Ambroggio E, Sorre B, Bassereau P, Goud B, Manneville JB, Antonny B: ArfGAPI generates an ArfI gradient on continuous lipid membranes displaying flat and curved regions. EMBO J 2010, 29:292-303.

FI000 Factor 8

Evaluated by Catherine Jackson 04 Dec 2009

13. Bigay J, Gounon P, Robineau S, Antonny B: Lipid packing sensed by AfrGAPI couples COPI coat disassembly to membrane bilayer curvature. Nature 2003, 426:563-6.

FI000 Factor 14

Evaluated by Karin Romisch 09 Dec 2003, Alfred Wittinghofer 12 Mar 2004, Rainer Pepperkok 14 May 2004 